\title{
Evaluation of Hydrophilic Polymers and their Combinations in Formulation of Sustained-Release Matrix Tablets of Water-Soluble Drug
}

\author{
B. S. Manjula, A. Srinatha* and B. K. Sridhar \\ Department of Pharmaceutics, National College of Pharmacy, Shimoga, India
}

\begin{abstract}
Aim of the work: The present study was aimed to investigate matrix-forming property of gum olibanum for sustained-release tablets of tramadol, a freely water-soluble drug. The possible synergistic effect of gum olibanum with hydroxypropyl methylcellulose (HPMC) and xanthan gum, and widely used pharmaceutical excipients on tablet properties was investigated. Methods: Matrix tablets were prepared containing polymers, either alone or in combinations, by wet granulation. Tablets were evaluated for their physico-mechanical properties, drug release and hydration properties. Results: Tablets swelling increased till $\sim 3-4 \mathrm{~h}$ this was followed by a decline phase. Drug release from the matrix tablets was dependent on the concentration of gum olibanum. Formulation with drug: gum olibanum in 1:2 released $94.37 \pm 2.37 \%$ drug in $6 \mathrm{~h}$. The drug release data was analyzed by model dependent and model independent equations. Combination of gum olibanum with HPMC or xanthan gum matrices significantly $(p<0.05)$ modulated drug release, which was reflected by the mean dissolution times (MDTs). Incorporation of lactose induced faster drug release compared to dicalcium phosphate and microcrystalline cellulose. Drug release largely followed first-order kinetics and non-Fickian type of diffusion.
\end{abstract}

Keywords: Diffusion, excipients, hydrophilic, similarity factor, gum olibanum

\section{INTRODUCTION}

Tramadol $\mathrm{HCl}$ is a water-soluble opioid analgesic drug used to relieve pain. It is used when other category drugs such as non-steroidal anti-inflammatory drugs or COX-2 inhibitors fail to relieve pain in patients. ${ }^{1}$ It is administered at a dose of $50-100 \mathrm{mg}$ and has a biological half-life of $\sim 5.5 \mathrm{~h}^{2}$ On oral administration, tramadol is rapidly absorbed with high bioavailability and excreted by urine. ${ }^{3,4}$

Hydrophilic polymers are widely used in sustained-release matrix formulations and the interest in the application of these polymers for prolonging drug release has increased. ${ }^{5,6}$ The popularity of these polymers is attributable to their swelling capabilities as well as their ability for direct compression. ${ }^{7}$ Natural gums, such as karaya gum, xanthan gum, and guar gum, are the most widely used hydrophilic polymers for the design of matrix tablets because of their cost-effectiveness and regulatory acceptance. Other than the above polymers, there are several natural polymers investigated for their application in sustained drug delivery. ${ }^{6}$ They form viscous gels in the presence of water, regardless of whether they are used alone or in combination with other gums or polymers, and are considered good matrixforming materials for sustained-release tablets. $^{8-11}$ Drug release from hydrophilic matrices occurs through dissolution, diffusion, erosion, or a combination of these mechanisms. ${ }^{12}$ Combination of different gums or polymers to increase matrix viscosity and optimize release often leads to synergistic interactions. ${ }^{6,12}$ Ideally, oral matrix formulations should contain polymers that
DOI: $10.5530 /$ ijper.48.3.7

Address for correspondence: Dr. A. Srinatha Department of Pharmaceutics, National College of Pharmacy, Shimoga, INDIA Phone: +91-9886446772.

E-mail: asrinatha@gmail. com; asrinatha@yahoo.com

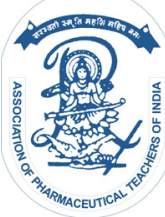

www.ijper.org 
yield steady zero-order release kinetics over an extended time period. ${ }^{13-15}$

Gum olibanum, a natural resin is obtained from a variety of species of Boswellia. It consists primarily of acid gum resin (56-60\%), carbohydrates $(30-36 \%)$ and volatile oil (3-8\%). The resin is composed of a resin acid (boswellic acid) and resene (olibamoresena) in equal proportions. ${ }^{16}$ Chowdary et $\mathrm{a}^{16}$ reported matrix forming ability of resin of gum olibanum using diclofenac as model drug. But study was limited and the formulation variables were not investigated. In another study, Chowdary et al ${ }^{17}$ evaluated use of gum olibanum in micro-encapsulation of nifedipine. Resin coated microcapsules exhibited a high encapsulation efficiency and drug release was by non-Fickian mechanism. Silver nanoparticles synthesized with aqueous extract of gum olibanum was reported to be stable as a water soluble component of olibanum serves as both reducing and stabilizing agent. ${ }^{18}$

The major objectives of this study were: (i) to evaluate the use of gum olibanum in sustained-release matrix tablets of tramadol, (ii) to comparatively evaluate gum olibanum with commonly used polymers HPMC and xanthan gum, and (iii) to study the influence of commonly used pharmaceutical excipients on gum olibanum tableting properties.

\section{MATERIALS AND METHODS}

\section{Materials}

Tramadol was a generous gift of Lupin laboratories ltd, Pune, India. Gum olibanum was procured commercially, dried at $60{ }^{\circ} \mathrm{C}$ and powdered in a ball mill.
Microcrystalline cellulose (Avicel ${ }^{\circledR}$ PH 101), lactose $\left(\right.$ Fast Flo $^{\circledR}$ ) and dibasic calcium phosphate, dehydrate $\left(\right.$ Emcompress $^{\circledR}$ ), were purchased from Sd-Fine chem, Mumbai, India. All other chemicals were of analytical grade and used as received. Commercial sustained release tablet of tramadol, Tramzac-TC (Zydus Cadila, India), was purchased commercially for comparative dissolution analysis.

\section{Preparation of matrix tablets}

Tablets were prepared by wet granulation method. Drug, polymer and excipients were passed through sieve \#80 before use. A physical mixture of drug and polymer(s) (Table 1) was suitably wetted with PVP K30 in isopropyl alcohol $(10 \% \mathrm{w} / \mathrm{v})$. Wet mass was sieved to obtain granules, dried at $40{ }^{\circ} \mathrm{C}$ for $2 \mathrm{~h}$. The dried granules were mixed with talc, magnesium stearate and compressed on a single station tableting machine (Cadmach machinery co. Pvt, Ltd Ahmadabad, India) using flat faced 12.60 $\mathrm{mm}$ punches.

\section{Micromeritics of granules}

The granules were evaluated for micromeritic properties like bulk density, tapped density, compressibility index, Hausner ratio, angle of repose by using standard procedures. All studies were carried out in triplicate $(n=3)$.

\section{Weight variation and hardness test}

For weight variation study, twenty tablets were selected randomly and average weight was recorded. Individual weight of the tablets was compared with the average weight to record difference between the weights. Hardness of tablets was determined using Monsanto hard-

\begin{tabular}{|c|c|c|c|c|c|c|c|c|c|c|c|c|c|c|}
\hline \multirow{2}{*}{$\begin{array}{l}\text { Ingredient } \\
\text { (mg/tablet) }\end{array}$} & \multicolumn{14}{|c|}{ Formulations } \\
\hline & F1 & F2 & F3 & F4 & F5 & F6 & F7 & F8 & F9 & F10 & F11 & F12 & F13 & F14 \\
\hline Tramadol & 100 & 100 & 100 & 100 & 100 & 100 & 100 & 100 & 100 & 100 & 100 & 100 & 100 & 100 \\
\hline Olibanum Gum & 200 & 300 & 400 & - & 200 & 280 & 120 & - & 200 & 280 & 120 & 300 & 300 & 300 \\
\hline HPMC K15M & - & - & - & 400 & 200 & 120 & 280 & - & - & - & - & - & - & - \\
\hline Xanthan Gum & - & - & - & - & - & - & - & 400 & 200 & 120 & 280 & - & - & - \\
\hline Fast $\mathrm{Flo}^{\circledR}$ & - & - & - & - & - & - & - & - & - & - & - & 100 & - & - \\
\hline Emcompress $^{\circledR}$ & - & - & - & - & - & - & - & - & - & - & - & - & 100 & - \\
\hline Avicel PH 101 & - & - & - & - & - & - & - & - & - & - & - & - & - & 100 \\
\hline
\end{tabular}

Batch size 40 tablets. Each tablet contained 1\% w/w of talc and magnesium stearate. 
ness tester by using 10 tablets from each formulation batch.

\section{Friability}

Ten tablets from each batch was weighed (Wo) and placed in Roche friabilator. The tablets were tumbled for 100 revolutions at $25 \mathrm{rpm}$. Tablets were de-dusted and the weight $(\mathrm{Wt})$ of tablets was recorded. The friability $(\%)$ of the formulations was calculated using the following equation:

$$
\text { Friability }(\%)=\frac{W o-W t}{W o} \times 100
$$

\section{Drug content}

Drug content in the formulations was estimated by extracting the drug from the powdered tablet using distilled water. Briefly, 10 tablets were powdered in a glass mortar and a powder quantity equal to the weight of a tablet was transferred to volumetric flask. Drug was extracted in $50 \mathrm{~mL}$ of distilled water with continuous shaking on a rotary shaker (Remi instruments, Mumbai, India) at $100 \mathrm{rpm}$ for $24 \mathrm{~h}$. The drug content in extracted fluid was analyzed using a UV-Spectrophotometer (UV1601, Shimadzu, Japan) at $271 \mathrm{~nm}$ against suitable blank.

\section{Swelling and erosion studies}

Pre-weighed tablets with wire mesh basket were placed in dissolution medium (distilled water). At different time intervals weight of the swollen tablet was recorded after wiping off excess of water. The swelling index (\%) was calculated by using the following equation.

$$
\text { Swelling Index }(\%)=\left(\frac{W t-W o}{W o}\right) 100
$$

Where, $W_{o}$ - initial weight of tablet and $W_{t}$ - weight of the tablet at time ' $t$ '.

Erosion studies were performed by using dissolution test apparatus 1, rotated at $50 \mathrm{rpm}$ with $900 \mathrm{~mL}$ of distilled water at $37 \pm 0.5^{\circ} \mathrm{C}$. The tablets were placed in wire mesh baskets and the weight was noted. At predefined time a basket containing the matrix tablet was removed and dried at $75{ }^{\circ} \mathrm{C}$ for $24 \mathrm{~h}$. The baskets were reweighed and the process was repeated until a constant weight was noted. The percentage of erosion was calculated using the equation:

$$
\text { Erosion }(\%)=\frac{\text { Initial weight }- \text { Final weight }}{\text { Initial weight }} \times 100
$$

\section{In vitro drug release}

Drug release studies were performed using six station USP-23 dissolution test apparatus (Tab machines,
Mumbai, India) with paddle speed of $50 \mathrm{rpm}$. The sink condition was maintained using $900 \mathrm{~mL}$ of freshly distilled water maintained at $37 \pm 0.5{ }^{\circ} \mathrm{C}$. At identified time intervals, an aliquot of $5 \mathrm{~mL}$ was withdrawn and replenished with fresh medium. The samples were filtered through Whatmann filter paper No 1 and the amount of drug in each aliquot was assayed on a UVSpectrophotometer (UV 1601, Shimadzu, Japan) at 271 $\mathrm{nm}$ using a suitable blank. All trials were conducted in triplicate and the average $( \pm$ S.D) reading was noted.

\section{Spectrometric analysis}

The UV-Spectrophotometric method for tramadol was validated at $271 \mathrm{~nm}$. The calibration curve in triplicate was constructed in water in the working range of 2-20 $\mu \mathrm{g} / \mathrm{mL}$. The prepared calibration curve was linear with a slope of 0.006 and regression coefficient of 0.9990 . None of the additives used in the formulation had any effect on the estimation of tramadol in the working concentration range.

\section{Release data analysis}

In vitro drug release data were fit to various models to analyze drug release kinetics and mechanism of drug release from the matrix tablets. For elucidating the kinetics of drug release, drug release data were fit to: zero order kinetics model, $Q=Q_{0}+k$ t; first order kinetics model, ${ }^{19} Q=Q_{0} e^{-k t}$ and Higuchi's square root model $^{20}$ $Q=K \sqrt{ }$. Where, $Q$ is the amount of drug released in time " $P$ ". $Q_{0}$ is the initial amount of drug in dissolution medium, $K$ is the release constant of respective equations, and $t$ is the release time.

In order to understand the drug release mechanism from the matrix tablets, data were fit to KorsmeyerPeppas empirical power law equation. ${ }^{21}$

$$
\frac{M t}{M \infty}=k t^{n}
$$

Where, $M_{t} / M_{\infty}$ is the fraction of drug released at time ' $t$, $K$ is the structural and geometrical constant and ' $n$ ' is the release exponent. For matrix tablets, when $n<0.45$ drug release is considered to be by diffusion while 0.45 $<\mathrm{n}<0.89$, is consider to release drug by a combination of drug diffusion as well as polymeric chain relaxation. When ' $n$ ' is 0.89 , the drug release is considered to show linearity towards zero order release kinetics and $n>0.89$ is indicative of super case II transport.

\section{Model dependent and independent analysis Weibull model:}

Dissolution data were also fit to Weibull model to further elucidate mechanism of release. Weibull equation expresses the accumulated fraction of the drug, $m$, in solution at time, ' $t$, by 


$$
\frac{M t}{M \infty}=1-\exp \left[\frac{-(t-T i)^{b}}{a}\right]
$$

In this equation, the scale parameter, ' $a$ ', defines the time scale of the process. The location parameter, $T$, represents the lag time before the onset of the dissolution or release process and in most cases will be zero and ' $b$ ' is the shape parameter. ${ }^{22,23}$

\section{Mean dissolution time}

Mean dissolution time (MDT) is used to characterize the drug release rate from a dosage form and indicates the drug release retarding efficiency of the polymer. MDT was calculated by using the following equation:

$$
M D T=\frac{\sum_{i=1}^{i=n} t_{m i d} \Delta M}{\sum_{i=1}^{i=n} \Delta M}
$$

Where ' $?$ ' is the dissolution sample number, ' $n$ ' is the number of dissolution sample time, ' $t$, ', is the time at the midpoint between ' $~ '$ and ' $i-1$ ', and ' $\Delta M$ ' is the amount of drug dissolved between ' $i$ ' and ' $i-1$ '.

\section{Similarity factor}

The similarities between dissolution profiles of marketed product and test formulations were determined by model independent procedure such as similarity factor $(f 2)$.

$$
f 2=50 \log \left\{\left[1+\left(\frac{1}{n}\right) \sum_{t=1}^{n}\left(R_{t}-T_{t}\right)^{2}\right]^{-0.5} \times 100\right\}
$$

Where ' $n$ ' is the number of pull points, ' $R t$ is the reference profile at time point ' $t$, and ' $T t$ ' is the test profile at the same time point.

\section{Statistical analysis}

The obtained dissolution data was statistically analysed by one-way ANOVA followed by Tukey method. A probability value of $p<0.05$ was considered as statistically significant.

\section{RESULTS}

\section{Micromeritics and physical properties}

The bulk and tapped densities of prepared granules was in the range of $0.245 \pm 0.02 \mathrm{~g} / \mathrm{cc}$ to $0.404 \pm 0.03 \mathrm{~g} / \mathrm{cc}$ and $0.269 \pm 0.01 \mathrm{~g} / \mathrm{cc}$ to $0.455 \pm 0.03 \mathrm{~g} / \mathrm{cc}$, respectively. Carr's compressibility index and Hausner ratio was less than $15 \pm 0.87 \%$ and $1.18 \pm 0.01$, respectively. Angle of repose $(\theta)$ for granules was in the range of $28.99 \pm 1.31$ to $36.79 \pm 1.76$.

\section{Weight variation and hardness}

Weight variation studies showed that the percentage deviation of the individual weight of tablets with average weight was $<5 \%$ which was within the acceptable limit. Hardness of the tablets ranged between $6.83 \pm$ 0.28 to $12 \mathrm{~kg} / \mathrm{cm}^{2}$. Tablets prepared with gum olibanum matrix exhibited a hardness of $7 \pm 0.29$ to $8.4 \pm$ $0.58 \mathrm{~kg} / \mathrm{cm}^{2}$. There was no significant $(p>0.05)$ change in the hardness of formulations F2 and F3. Tablets with HPMC matrix, either alone or in combination with gum olibanum (F4 to F7), showed highest hardness and was in the range of $10 \pm 0.5$ to $12 \pm 0.01 \mathrm{~kg} /$ $\mathrm{cm}^{2}$. Xanthan gum matrix tablets alone or in combination with gum olibanum (F8-F11) showed hardness of $8.50 \pm 0.5$ to $9.50 \pm 0.5 \mathrm{~kg} / \mathrm{cm}^{2}$. The results indicate that prepared tablets were having sufficient strength to withstand the mechanical stress. The observed results indicate of dependency of the hardness on the type of the polymer(s) present in the matrix with HPMC matrix tablets exhibiting highest hardness in comparison to other formulations. The hardness of olibanum matrix tablets $\left(8.4 \pm 0.58 \mathrm{~kg} / \mathrm{cm}^{2}\right)$ was influenced by the addition of excipients. It decreased significantly to $6.83 \pm$ 0.28 and $7.03 \pm 0.15 \mathrm{~kg} / \mathrm{cm}^{2}$ with addition of lactose and microcrystalline cellulose respectively while hardness increased to $9.10 \pm 0.17 \mathrm{~kg} / \mathrm{cm}^{2}$ on addition of dicalcium phosphate.

\section{Friability and drug content}

The friability and drug content of matrix tablet formulations was in the range of $0.09 \pm 0.01$ to $0.51 \pm 0.03 \%$ and $95.02 \pm 1.53$ to $103.9 \pm 3.88 \%$, respectively.

\section{Swelling and erosion studies}

Swelling index of the prepared formulations was studied in water for $6 \mathrm{~h}$. Swelling index was directly proportional to the concentration of the olibanum gum and as its concentration in the matrix was increased, swelling index increased proportionally (Figure 1).

All the formulations, except tablets with HPMC, exhibited a biphasic swelling phenomenon wherein the swelling increased till a period ( $\sim 3$ to $4 \mathrm{~h}$ ) after which the swelling decreased (Figure 2, 3). But tablets with HPMC matrix (F4) were exception to this phenomenon wherein swelling increased with time and decline phase was not observed during the study period. In tablets with combinations of polymers, the tablets with low concentration of HPMC (high gum olibanum matrix) exhibited low swelling index.

The erosion was higher in gum olibanum matrix in comparison to xanthan gum HPMC matrices. At the end of $24 \mathrm{~h}$ erosion study, $74.35 \pm 1.25 \%$ of gum olibanum matrix eroded while during the same period $39.20 \pm$ 


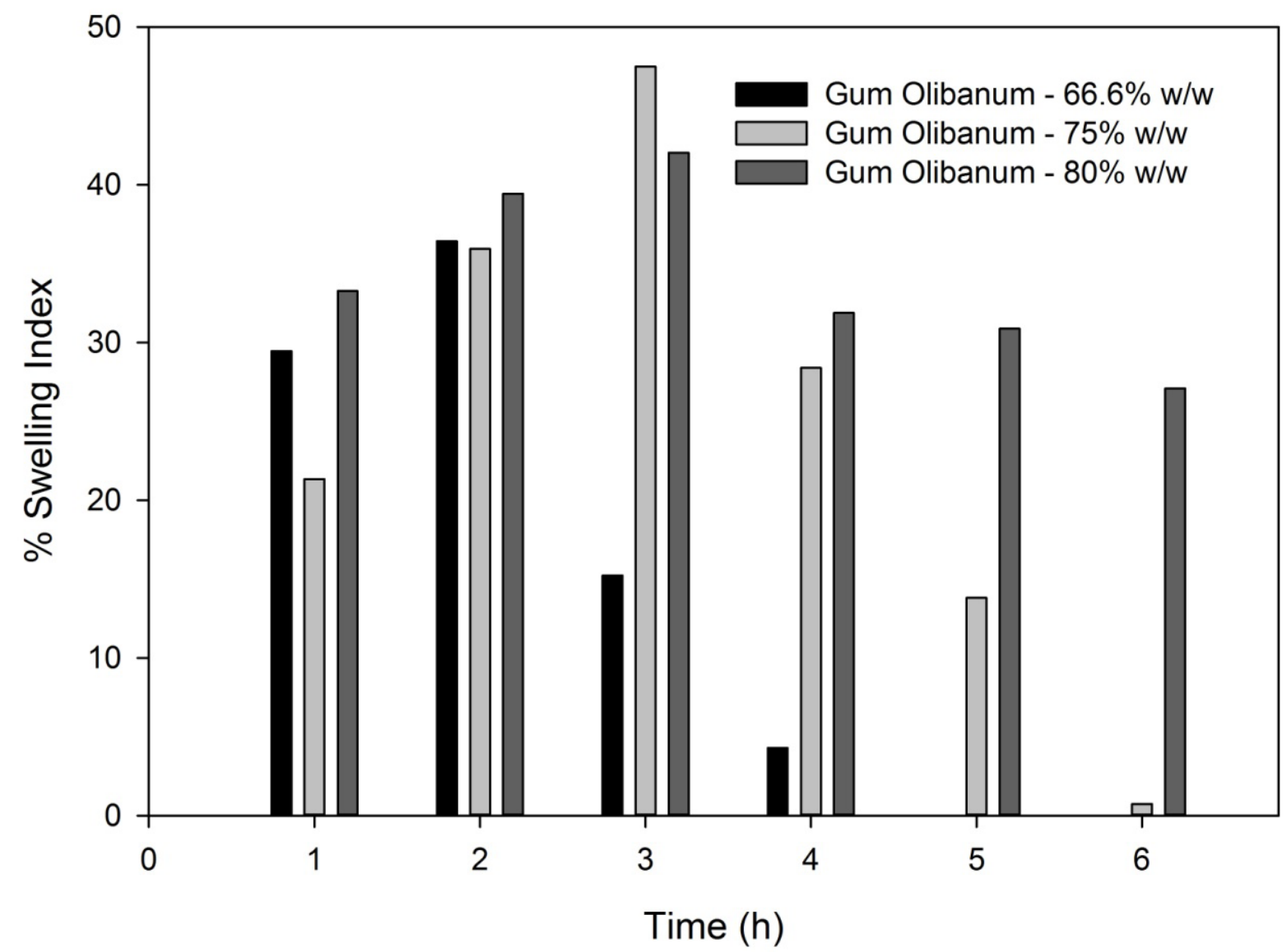

Figure 1: Graph showing swelling index with time of matrix tablets prepared with gum olibanum

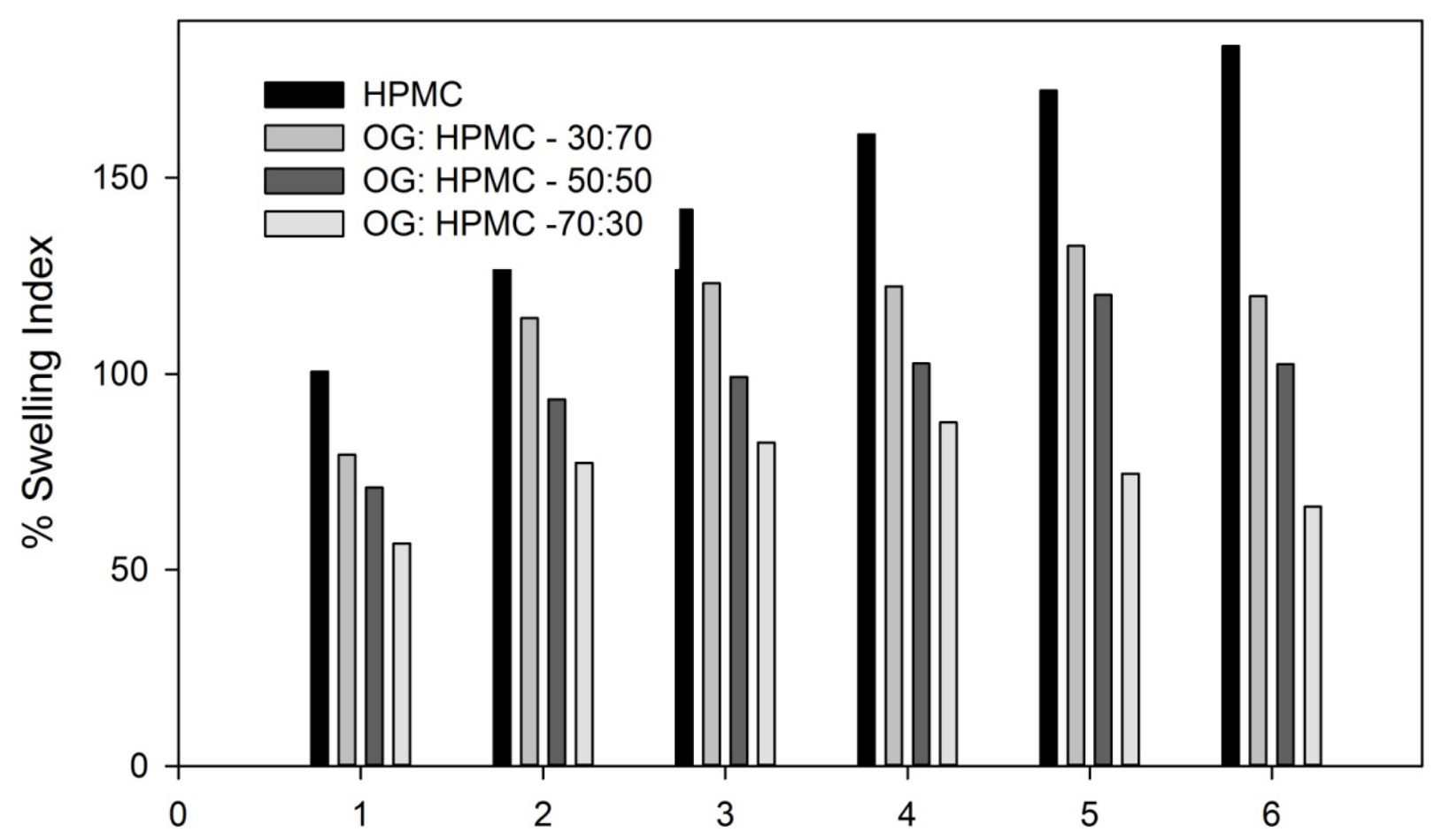

Figure 2: Graph showing swelling index with time of matrix tablets prepared with combination of HPMC and gum olibanum 


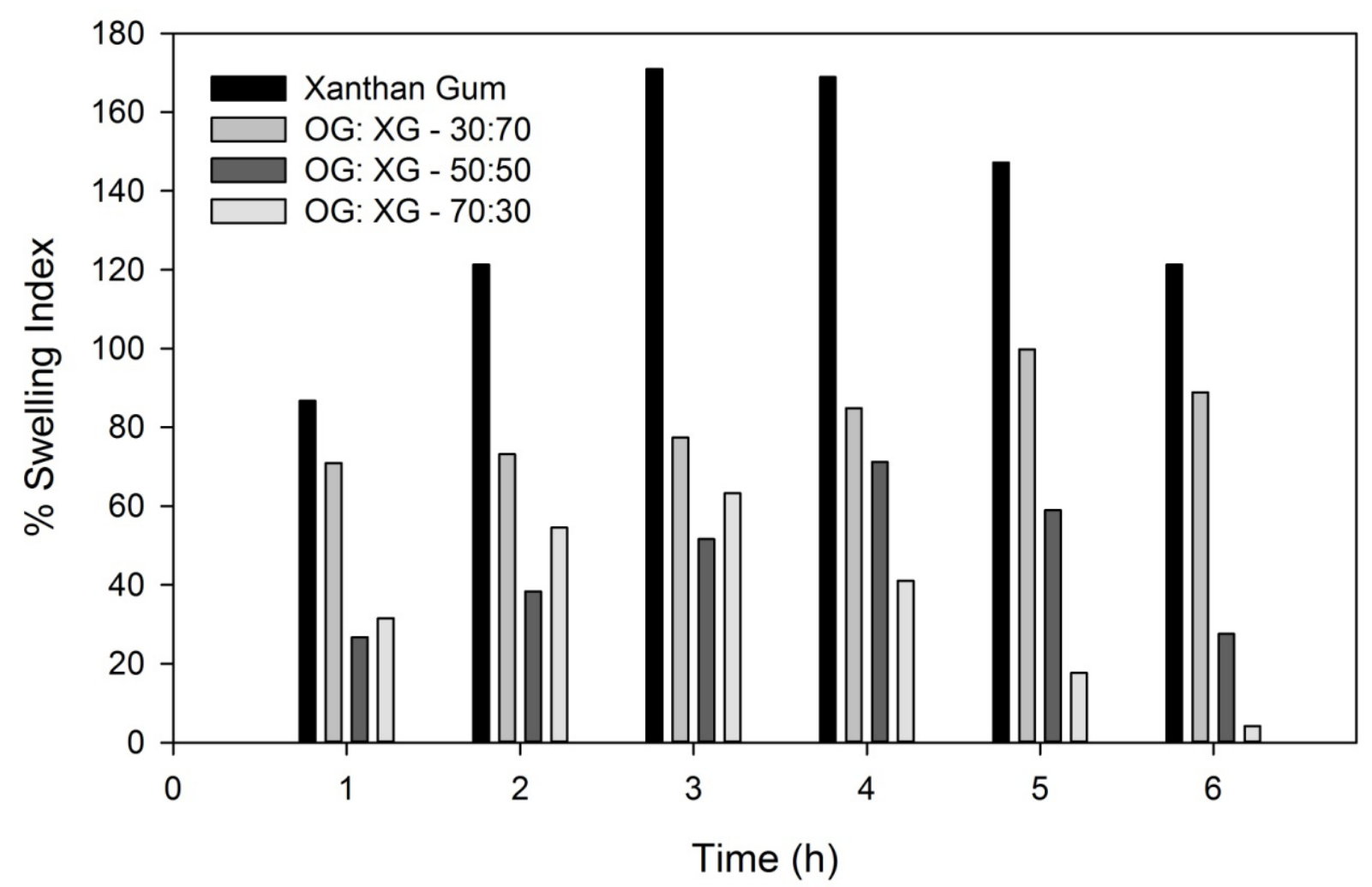

Figure 3: Graph showing swelling index with time of matrix tablets prepared with combination of xanthan gum and gum olibanum

$2.94 \%$ and $55.28 \pm 2.01 \%$ of HPMC and xanthan gum matrix eroded respectively. In case of matrix tablets in combinations of gum olibanum with HPMC or xanthan gum, the erosion increased with increase in concentration of gum olibanum in matrix (data not shown).

\section{In vitro drug release studies}

\section{Effect of polymers on drug release}

In vitro drug release profiles of formulations and market product are shown in figures 4 to 8 . Formulations containing gum olibanum, HPMC and xanthan gum released $85.15 \pm 1.52 \%, 62.42 \pm 7.57 \%$ and $73.47 \pm$ $1.35 \%$ of drug respectively, during $8 \mathrm{~h}$ of study indicating that the polymer concentration in each formulation sustains drug release. In formulations containing HPMC or xanthan gum, either alone or in combination with gum olibanum, the ratio of drug to polymer was kept constant at 1:4. Formulation F1 (drug:olibanum $1: 2)$ showed higher drug release with $94.37 \pm 2.37 \%$ in 6 h. However, significantly $(p<0.05)$ slower drug release was observed for formulations F2 (drug:olibanum - 1:3) and F3 (drug:olibanum - 1:4) during the same period of dissolution study (figure 4). The drug release from these formulations was easily modulated with inclusion of gum olibanum in the polymeric matrix. The drug release from the matrix decreased with the increased concentration of gum olibanum. Drug release from the polymeric matrices consisting of mixture of polymers (HPMC or xanthan gum with gum olibanum) was dependent on the proportion of gum olibanum.

\section{Effect of excipients on drug release}

Formulations with commonly used excipients of different nature were studied to investigate the influence of the excipients on drug release (figure 8). Formulation with drug: olibanum (1:4) was incorporated with Fast Flo $^{\circledR}$ (lactose, F12), Emcompress ${ }^{\circledR}$ (Dicalcium phosphate, F13) and Avicel PH $101^{\circledR}$ (Microcrystalline cellulose, F14). Formulations prepared with these excipients showed a burst release of $\sim 37-40 \%$ in $1 \mathrm{~h}$ irrespective of the nature of the excipient. In case of formulation with Fast $\mathrm{Flo}^{\circledR}$, drug release was significantly $(p<0.05)$ faster in comparison to formulations containing other two excipients. In comparison to matrix tablets with Emcompress ${ }^{\circledR}$, tablets prepared with Avicel PH $101^{\circledR}$ exhibited faster drug release. Except formulation comprising Fast Flo ${ }^{\circledR}$ other two formulation showed a prolong release of drug up to $8 \mathrm{~h}$ while the former prolonged release up to $6 \mathrm{~h}$.

\section{Model dependent and independent analysis}

The prepared matrix tablets followed first order kinetics with a regression co-efficient of 0.8697- 0.9954, except formulations F12 and F13 which followed zero order kinetics $\left(\mathrm{r}^{2}=0.9544\right.$ and 0.8925 , respectively). 


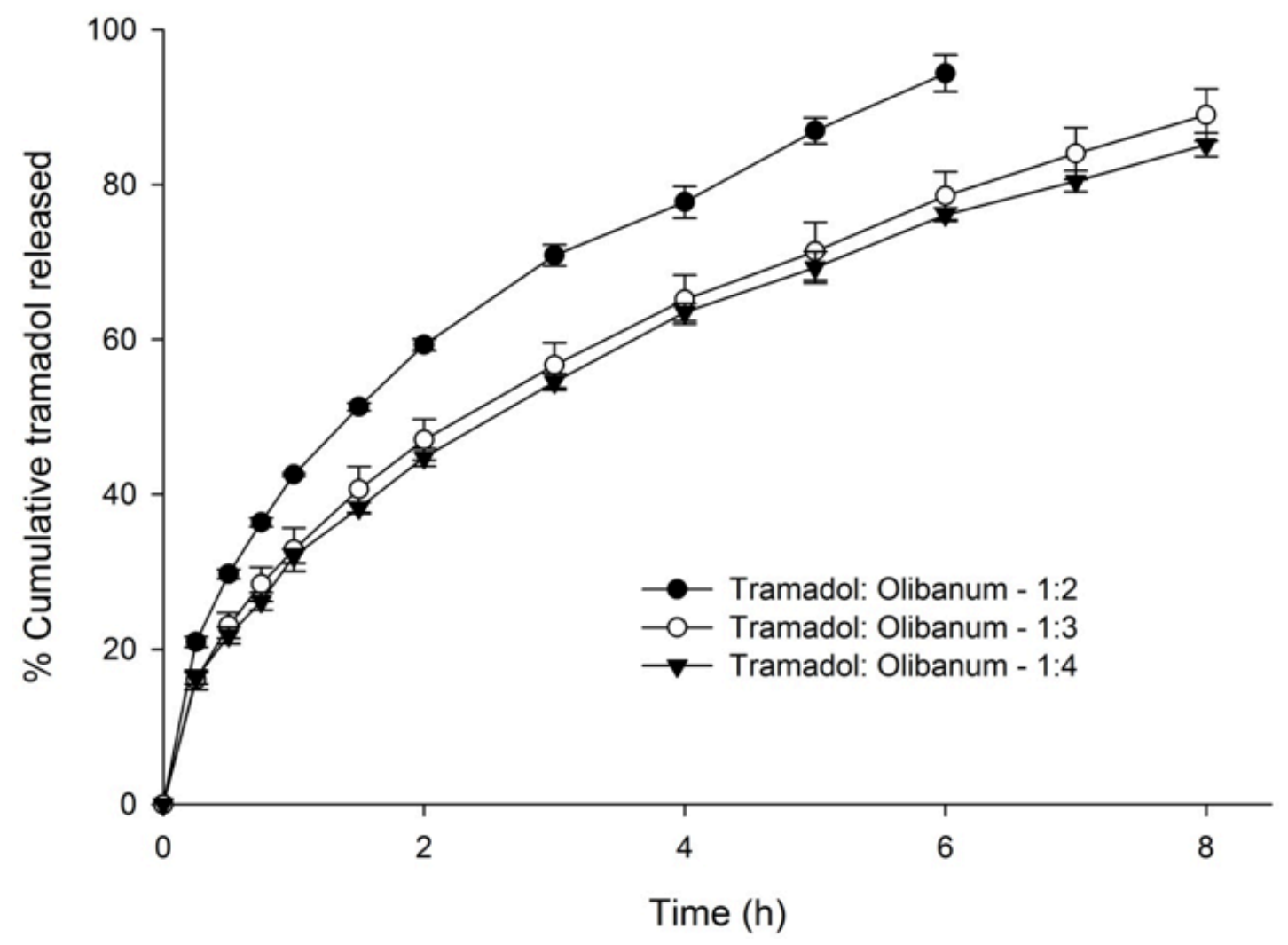

Figure 4: Effect of various concentration of gum olibanum on tramadol release from tablets

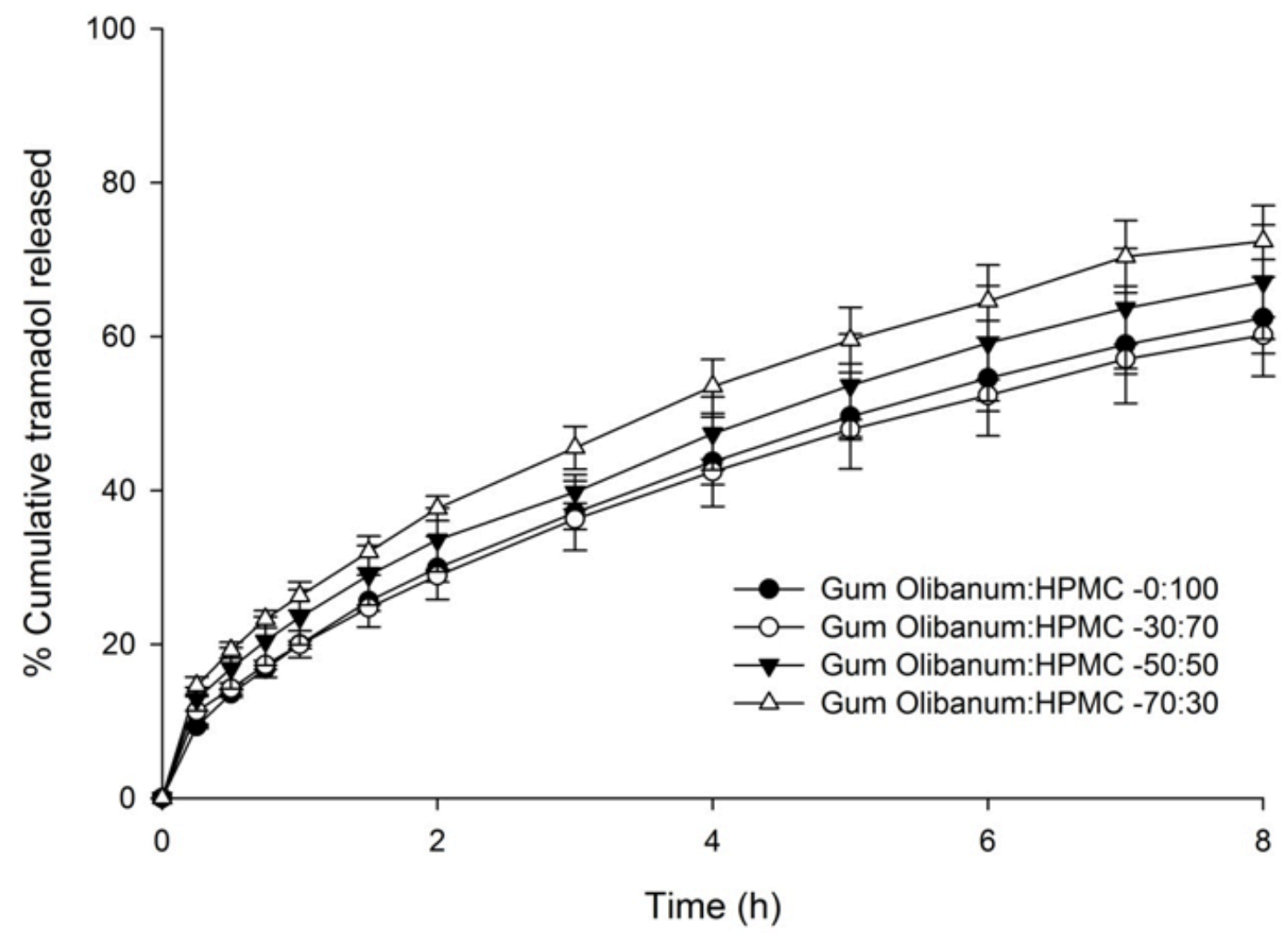

Figure 5: Effect of combination of HPMC and gum olibanum on tramadol release from tablets 


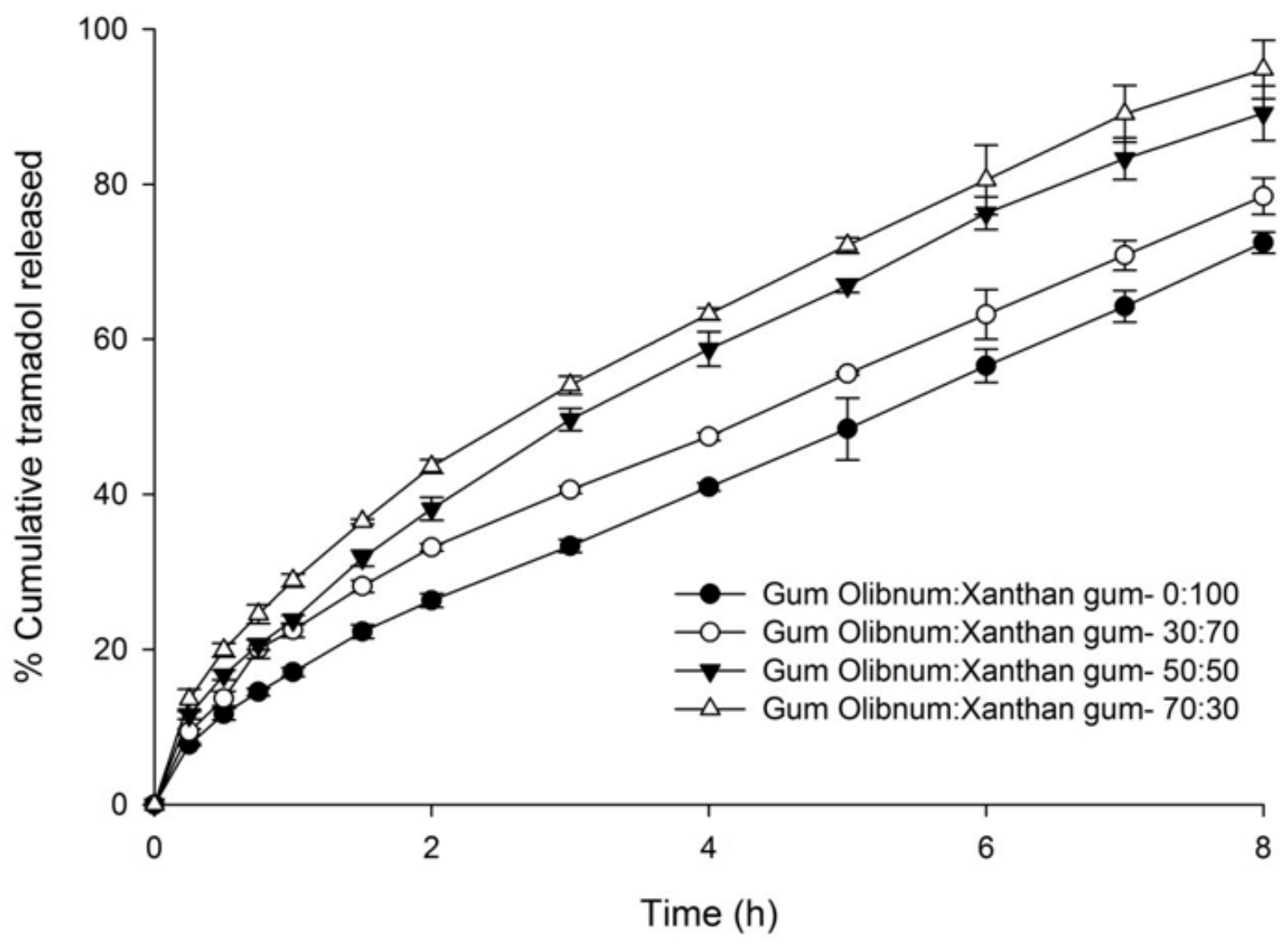

Figure 6: Effect of combination of xanthan gum and gum olibanum on tramadol release from tablets

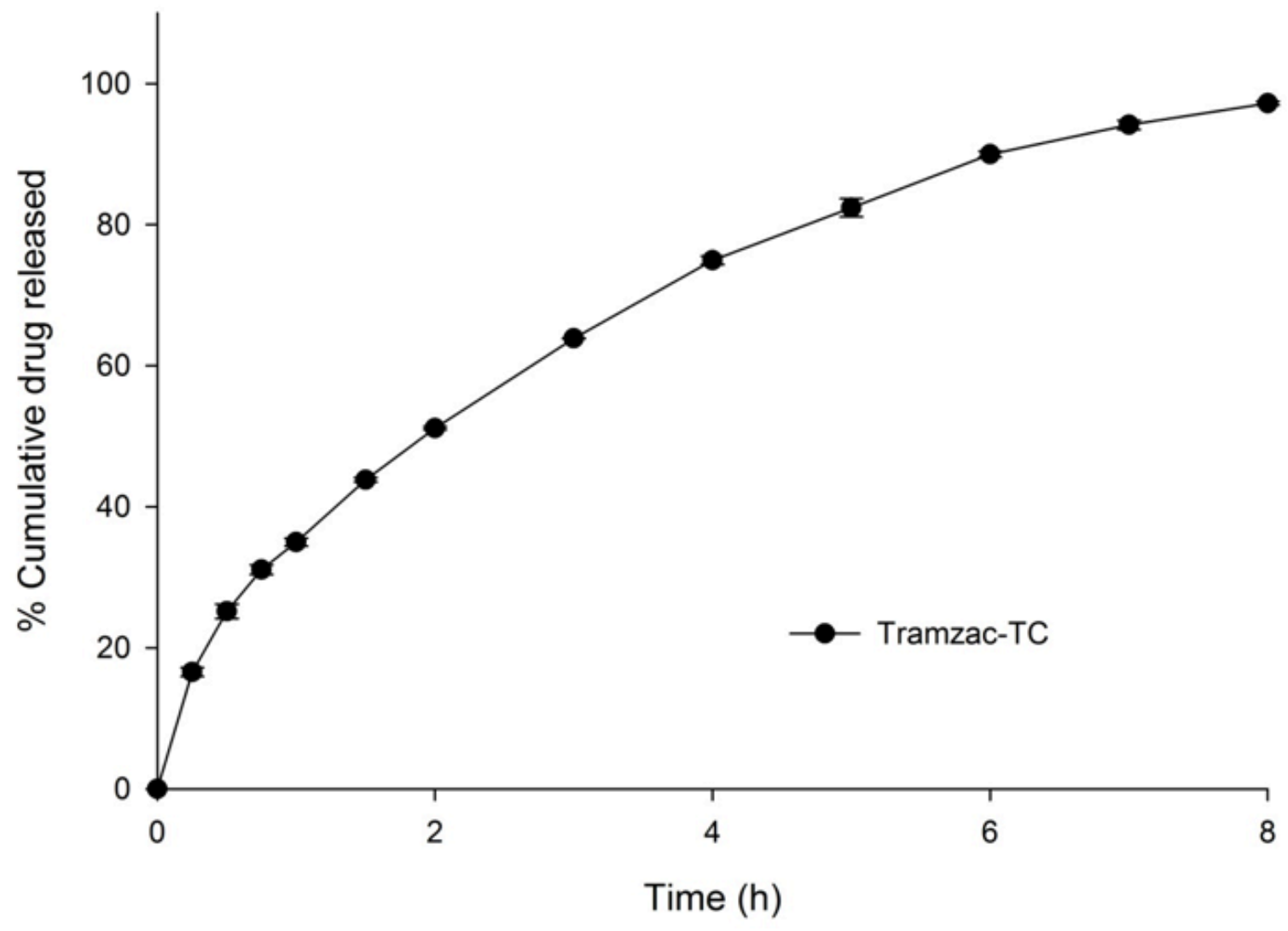

Figure 7: Drug release profile of commercial product (Tramzac-TC) 


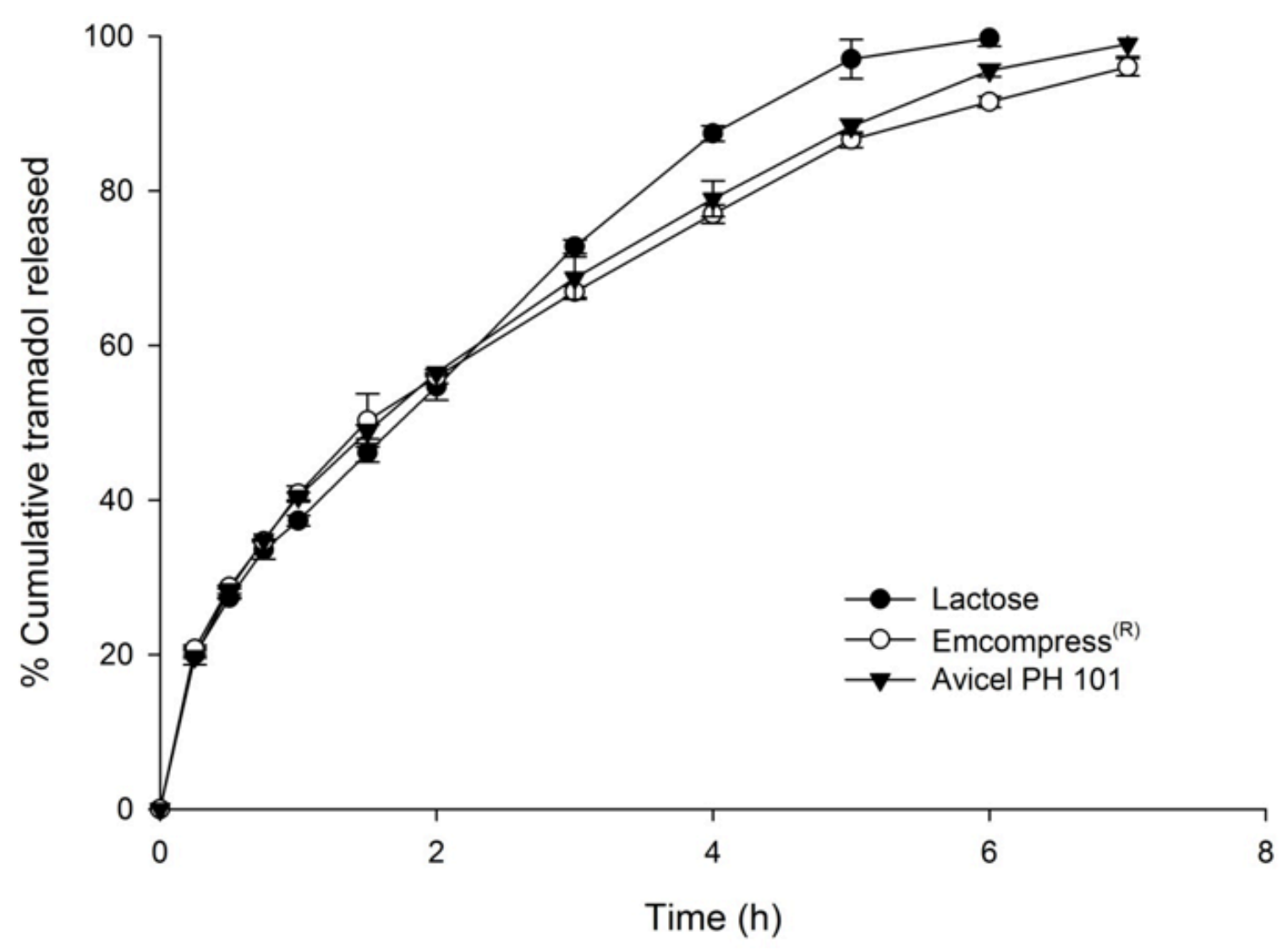

Figure 8: Effect of commonly used excipients on drug release from gum olibanum tablets

The data from dissolution studies was further fit to Higuchi's equation to analyze drug release mechanism. The regression co-efficient values of Higuchi's model indicated that formulation exhibit linearity towards diffusion mechanism with a regression values in the range of 0.9599-0.9991. Further data treatment using Korsmeyer-Peppas equation indicated that drug release mechanism from the prepared matrices followed anomalous diffusion mechanism or non-Fickian (Table 2).

\section{DISCUSSION}

The micromeritic parameter compressibility index represents packing arrangement of powder. It indicates the possibility of consolidation of the granules and powders during tableting, shipping, transportation and handling. ${ }^{24}$ Its values can best be used for assessing the flow properties of the granules and powders. Compressibility index values determined for the granules indicated good flow property which was further supported by the Hausner ratio and angle of repose of granules.

The results of friability studies further supported the ability of the prepared matrix tablets to withstand possible mechanical impact during various steps from manufacturing until final usage. The swelling index or hydration of xanthan gum matrix tablets increased till $4 \mathrm{~h}$ after which hydration decreased with time. At this stage tablets lost the shape and further contact with water made the tablet to break due to erosion. The initial increase and subsequent decrease in swelling index was probably due to the erosion of surface layer of matrix tablet. In formulations with combination of gum olibanum and xanthan gum, swelling was dependent on the concentration of xanthan gum. Higher concentration of xanthan gum in the matrix exhibited higher swelling index.

Matrix tablets prepared with gum olibanum exhibited sustained release of tramadol. When used in low concentration (drug:polymer - 1:1) complete drug release was achieved in $6 \mathrm{~h}$ of dissolution study. Increasing the concentration of gum olibanum resulted in more sustained effect and drug release was extended up to $8 \mathrm{~h}$. At a low concentration, gum olibanum matrix tablets exhibited a burst release of $42.56 \pm 0.23 \%$ in $1 \mathrm{~h}$. This observed burst release was decreased by $\sim 10 \%$ with increasing polymer concentration. Among the three polymers, cumulative drug release from HPMC polymeric matrix was slower in comparison to xanthan gum and gum olibanum. Surprisingly, MDT value of xanthan gum matrix tablets (214.4 min) was higher in comparison to HPMC matrix tablets (164.9 $\mathrm{min})$. The result was unexpected since higher amount of drug release is supported by smaller MDT. ${ }^{25}$ The observed deviation 
in MDT values could possibly related to hydration and erosion of polymeric matrix. The drug release in xanthan gum matrix tablets was slower in comparison to HPMC matrix up to $4 \mathrm{~h}$ of dissolution studies. However, during the remaining $4 \mathrm{~h}$ dissolution studies, the xanthan gum matrix exhibited higher drug release compared to HPMC matrix tablets. As can be observed from figures 2 and 3, the hydration rate was higher in xanthan gum as compared with HPMC matrix. In xanthan gum matrix equilibrium swelling was attained in 4 $\mathrm{h}$ and subsequently swelling decreased causing disruption of the matrix. This possibly caused higher drug release from xanthan gum matrix in comparison to HPMC matrix tablets. Drug release from gum olibanum matrix was dependent on its concentration in the tablet wherein drug release reduced with increase in gum olibanum concentration. The difference in drug release between formulations is further supported by MDT values wherein F1 exhibited a lower MDT (112.5 min) than F2 and F3 (Table 2). Similar release profile for diclofenac sodium using resin extracted from gum olibanum was reported by Chowdary et al. ${ }^{16}$
With increase in the concentration of gum olibanum in the matrix prepared with combinations of polymers, drug release increased. This observed phenomenon is best explained on the basis of the hydrophilic properties of the polymers. HPMC and xanthan gum represents hydrogels, a class of hydrophilic polymers. Both the polymers have been widely reported for their matrix forming properties and controlling the drug release rate. Gum olibanum is a resinous polymeric matrix material with more than $50 \%$ resin content. The ability of gum olibanum to swell on contact with moisture is less compared to HPMC and xanthan gum. As the concentration of gum olibanum in the matrix was increased the swelling index decreased indicating its inability to form hydrogel matrix. Similarly, drug release from the matrix was also affected by the concentration of gum olibanum in the matrix. Varshosaz et $\mathrm{al}^{25}$ observed that inclusion of xanthan gum and guar gum has significantly modified tramadol release from HPMC matrix tablets. When two or more polymers are used in a matrix, if they form a semi- or complete interpenetrating network, it form a rigid matrix resulting in slower drug release. But in the

Table 2: Model dependent and independent analysis parameters

\begin{tabular}{|c|c|c|c|c|}
\hline Formulation & $\begin{array}{c}\text { Release } \\
\text { exponent (n) }\end{array}$ & Weibull factor (b) & f2 & MDT (min) \\
\hline $\mathrm{F} 1$ & 0.46 & 0.67 & 62.45 & 112.5 \\
\hline $\mathrm{F} 2$ & 0.49 & 0.65 & 75.12 & 152.9 \\
\hline F3 & 0.49 & 0.63 & 68.58 & 149.1 \\
\hline $\mathrm{F} 4$ & 0.56 & 0.74 & 41.26 & 164.9 \\
\hline F5 & 0.49 & 0.55 & 47.39 & 159.1 \\
\hline F6 & 0.48 & 0.57 & 53.84 & 150.1 \\
\hline $\mathrm{F} 7$ & 0.51 & 0.76 & 42.02 & 162.7 \\
\hline F8 & 0.65 & 0.81 & 38.36 & 214.4 \\
\hline F9 & 0.62 & 0.75 & 51.71 & 166.2 \\
\hline F10 & 0.57 & 0.73 & 60.72 & 173.3 \\
\hline F11 & 0.56 & 0.75 & 43.77 & 179.1 \\
\hline F12 & 0.54 & 0.77 & 71.31 & 110.2 \\
\hline F13 & 0.47 & 0.66 & 66.12 & 123.9 \\
\hline F14 & 0.47 & 0.69 & 69.19 & 115.5 \\
\hline Tramzac TC & 0.53 & 0.71 & - & 140.9 \\
\hline
\end{tabular}


present study, the matrix probably did not form interpenetrating network since HPMC and xanthan gum were highly swellable in comparison to gum olibanum. This possibly resulted in a matrix with increased porosity resulting in higher drug release from the matrix containing combination of HPMC or xanthan gum with gum olibanum. Increasing concentration of polymers seems to increase the path length for drug diffusion as a consequence of which drug release decreases.

The effect of commonly used pharmaceutical bulking agents lactose, MCC and DCP on physico-mechanical properties were studied. Drug release in formulations with lactose was faster in comparison to formulations prepared with DCP and MCC as bulking agents. The observed result was in the expected line. Lactose (Fast $\mathrm{Flo}^{\circledR}$ ) is water soluble and in contact with dissolution medium induces channeling effect within polymeric matrix, while Avicel PH $101^{\circledR}$ being water dispersible exhibited a marginal increase in drug release in comparison to formulation with an insoluble excipient Emcompress $^{\circledR}$. In a previous report by Yadav et $\mathrm{al}^{26}$ di-calcium phosphate in comparison to MCC showed faster venlafaxine release and complete drug release was observed within $3 \mathrm{~h}$ when used in HPMC matrix tablets. However, in case of cetyl alcohol the drug release from formulations containing DCP was prolonged than formulations containing MCC.

Tramadol release from the prepared matrix tablets followed either concentration dependent or independent kinetics, and non-Fickian mechanism. The observed non-Fickian mechanism of drug release was in contrast to the findings of Chowdary et $\mathrm{al}^{16}$ who reported a Fickian mechanism of drug release. This deviation could possibly related to the nature of polymer matrix. While Chowdary et $\mathrm{al}^{16}$ used extracted resin from gum olibanum as matrix in the present study untreated gum olibanum was evaluated. Change in solubility profile of drugs and nature of the resin alter the mechanism of drug release from the matrix tablets. The shape parameter (b) of Weibull equation characterizes drug release curve as exponential $(b=1)$, parabolic $(b>1)$ or initial slope followed with an exponential curve $(b<1)$. The determined shape parameter values was $<1$ indicating a drug release profile following higher initial slope followed with a consistent exponential curve.

Similarity factor $(f 2)$ was used to compare the release profiles of the prepared formulations with marketed product Tramzac-TC. As shown in table 2, only a few formulations showed similarity with marketed product with $f 2$ values above 50 . According to USFDA, two dissolution profiles are declared to be similar if calculated $f 2$ value is in the range of $50-100 .{ }^{22}$

\section{CONCLUSION}

Tramadol release from matrix tablets was influenced by the concentration of gum olibanum in the matrix. The drug release can be easily modulated by either adjusting the concentration of gum olibanum or by incorporation of other natural polymers. A judicious selection of pharmaceutical excipients is important since the drug release is significantly influenced by the properties of the used excipient.

\section{ACKNOWLEDGE}

Authors are thankful to Lupin laboratories, Pune, India for providing tramadol hydrochloride as gift sample.

\section{CONFLICT OF INTEREST}

Authors have no conflict of interest to declare.

\section{ABBRIVATION}

HPMC- Hydroxypropyl methylcellulose

MDT- Mean dissolution time

PVP - Polyvinyl pyrrolidone

MCC- Microcrystalline cellulose

DCP-Dicalcium phosphate

\section{REFERENCES}

1. Brandt KD. A critique of the 2000 update of the American college of rheumatology recommendations for management of hip and knee osteoarthritis. Arthritis Rheum. 2001; 44: 2451-5.

2. Sudha BS, Sridhar BK, Srinatha A. Modulation of tramadol release from a hydrophobic matrix: implications of formulations and processing variables. AAPS PharmSciTech. 2010; 11(1): 433-40.

3. Grond S, Sablotzki A. Clinical pharmacology of tramadol. Clin Pharmacokinet. 2004; 43: 879-923.

4. Raber M, Hofmann S, Junge K, Momberger H, Kuhn D. Analgesic efficacy and tolerability of tramadol $100 \mathrm{mg}$ sustained-release capsules in patients with moderate to severe chronic low back pain. Clin Drug Investig. 1999; 17(6): 415-23.

5. Al-Saidan SM, Krishnaiah YSR, Patro SS, Satyanaryana V. In vitro and in vivo evaluation of guar gum matrix tablets for oral controlled release of watersoluble diltiazem hydrochloride. AAPS PharmSciTech. 2005; 6(1): E14-21.

6. Sujja-areevath J, Munday DL, Cox PJ, Khan KA. Relationship between swelling, erosion and drug release in hydrophillic natural gum mini-matrix formulations. Eur J Pharm Sci. 1998; 6: 207-17.

7. Doelker E. Water swollen cellulose derivatives in pharmacy. In: Peppas NA, editor. Hydrogels Med Pharm. Vol II. Boca Raton, Florida: CRC Press; 1987. p. $115-60$

8. Dürig T, Fassihi R. Guar-based monolithic matrix systems: effect of ionizable and non-ionizable substances and excipients on gel dynamics and release kinetics. J Control Release. 2002; 80(1): 45-56.

9. Cox PJ, Khan KA, Munday DL, Sujja-areevath J. Development and evaluation of a multiple-unit oral sustained release dosage form for $\mathrm{S}(+)$-ibuprofen: preparation and release kinetics. Int J Pharm. 1999; 193(1): 73-84.

10. Hariharan M, Wheatley TA, Price JC. Controlled-release tablet matrices from carrageenans: compression and dissolution studies. Pharm Dev Technol. 1997; 2(4): 383-93. 
11. Prajapati VD, Jani GK, Moradiya NG, Randeria NP. Pharmaceutical applications of various natural gums, mucilages and their modified forms. Carbohydr Polym. 2013; 92(2): 1685-99.

12. Munday DL, Cox PJ. Compressed xanthan and karaya gum matrices: hydration, erosion and drug release mechanisms. Int J Pharm. 2000; 203(1): 179-92.

13. Krishnaiah YSR, Karthikeyan RS, Satyanarayana V. A three-layer guar gum matrix tablet for oral controlled delivery of highly soluble metoprolol tartrate. Int J Pharm. 2002; 241(2): 353-66.

14. Toti US, Aminabhavi TM. Modified guar gum matrix tablet for controlled release of diltiazem hydrochloride. J Control Release. 2004; 95: 567-77.

15. Tiwari SB, Murthy TK, Pai MR, Mehta PR, Chowdary PB. Controlled release formulation of tramadol hydrochloride using hydrophilic and hydrophobic matrix system. AAPS Pharm Sci Tech. 2003; 4(3): E31.

16. Chowdary KPR, Mohapatra P, Krishna NM. Evaluation of olibanum and its resin as rate controlling matrix for controlled release of diclofenac. Indian $\mathrm{J}$ Pharm Sci. 2006; 68: 497-500.

17. Chowdary KPR, Mohapatra P, Krishna NM. Evaluation of olibanum resin as microencapsulating agent for controlled drug delivery. Indian J Pharm Sci. 2006; 68: 461-4.

18. Kora AJ, Sashidhar RB, Arunachalam J. Aqueous extract of gum olibanum (Boswellia serrata): A reductant and stabilizer for the biosynthesis of antibacterial silver nanoparticles. Process Biochem. 2012; 47: 1516-20.
19. Wagner JG. Interpretation of percent dissolved-time plots derived fromin vitro testing of conventional tablets and capsules. J Pharm Sci. 1969; 58: 1253-7.

20. Higuchi T. Mechanism of sustained-action medication. Theoretical analysis of rate of release of solid drugs dispersed in solid matrices. J Pharm Sci. 1963; 52: 1145-9.

21. Korsmeyer RW, Gurny R, Doelker E, Buri P, Peppas NA. Mechanisms of solute release from porous hydrophilic polymers. Int J Pharm. 1983; 15(1): 25-35.

22. Costa P, Lobo JS. Modeling and comparison of dissolution profiles. Eur J Pharm Sci. 2001; 13(2): 123-33.

23. Langenbucher F. Linearization of dissolution rate curves by the Weibull distribution. J Pharm Pharmacol. 1972; 24(12): 979-81.

24. Carr RL. Evaluating flow properties of solids. Chem Eng. 1965;72(2):163-8.

25. Varshosaz J, Tavakoli N, Kheirolahi F. Use of hydrophilic natural gums in formulation of sustained-release matrix tablets of tramadol hydrochloride. AAPS Pharm Sci Tech. 2006; 7(1): E24.

26. Yadav K, Yadav D, Srivastava AK. Evaluation of hydrophilic, hydrophobic and waxy matrix excipients for sustained release tablets of Venlafaxine hydrochloride. Drug Dev Ind Pharm. 2013; 39(8): 1197-206. 